\title{
Reactive oxygen species generation mediated by NADPH oxidase and PI3K/Akt pathways contribute to invasion of Streptococcus agalactiae in human endothelial cells
}

\author{
Jessica Silva Santos de Oliveira', Gabriela da Silva Santos ${ }^{1}$, João Alfredo Moraes ${ }^{2}$, \\ Alessandra Mattos Saliba ${ }^{3}$, Thereza Christina Barja-Fidalgo ${ }^{2}$, \\ Ana Luíza Mattos-Guaraldi ${ }^{3}$, Prescilla Emy Nagao ${ }^{1 /+}$ \\ ${ }^{1}$ Universidade do Estado do Rio de Janeiro, Instituto de Biologia Roberto Alcântara Gomes, Departamento de Biologia Celular, \\ Laboratório de Biologia Molecular e Fisiologia de Estreptococos, Rio de Janeiro, RJ, Brasil \\ ${ }^{2}$ Universidade do Estado do Rio de Janeiro, Instituto de Biologia Roberto Alcântara Gomes, Departamento de Biologia Celular, \\ Laboratório de Farmacologia Celular e Molecular, Rio de Janeiro, RJ, Brasil \\ ${ }^{3}$ Universidade do Estado do Rio de Janeiro, Faculdade de Ciências Médicas, Rio de Janeiro, RJ, Brasil
}

BACKGROUND Streptococcus agalactiae can causes sepsis, pneumonia, and meningitis in neonates, the elderly, and immunocompromised patients. Although the virulence properties of $S$. agalactiae have been partially elucidated, the molecular mechanisms related to reactive oxygen species (ROS) generation in infected human endothelial cells need further investigation.

OBJECTIVES This study aimed to evaluate the influence of oxidative stress in human umbilical vein endothelial cells (HUVECs) during $S$. agalactiae infection.

METHODS ROS production during S. agalactiae-HUVEC infection was detected using the probe CM-H2DCFDA. Microfilaments labelled with phalloidin-FITC and p47phox-Alexa 546 conjugated were analysed by immunofluorescence. mRNA levels of p47phox (NADPH oxidase subunit) were assessed using Real Time qRT-PCR. The adherence and intracellular viability of $S$. agalactiae in HUVECs with or without pre-treatment of DPI, apocynin (NADPH oxidase inhibitors), and LY294002 (PI3K inhibitor) were evaluated by penicillin/gentamicin exclusion. Phosphorylation of p47phox and Akt activation by $S$. agalactiae were evaluated by immunoblotting analysis.

FINDINGS Data showed increased ROS production 15 min after HUVEC infection. Real-Time qRT-PCR and western blotting performed in HUVEC infected with $S$. agalactiae detected alterations in mRNA levels and activation of p47phox. Pre-treatment of endothelial cells with NADPH oxidase (DPI and apocynin) and PI3K/Akt pathway (LY294002) inhibitors reduced ROS production, bacterial intracellular viability, and generation of actin stress fibres in HUVECs infected with S. agalactiae.

CONCLUSIONS ROS generation via the NADPH oxidase pathway contributes to invasion of S. agalactiae in human endothelial cells accompanied by cytoskeletal reorganisation through the PI3K/Akt pathway, which provides novel evidence for the involvement of oxidative stress in S. agalactiae pathogenesis.

Key words: Streptococcus agalactiae - HUVEC - reactive oxygen species - NADPH oxidase - p47phox - PI3K/Akt pathway

Streptococcus agalactiae [group B Streptococcus $(\mathrm{GBS})]$ is found as a commensal in the gastrointestinal and the genitourinary tracts of up to $30 \%$ of healthy adults. Although $S$. agalactiae is a significant cause of neonatal meningitis and septicaemia (Verani et al. 2010), cases of invasive infections have been increasingly reported in elderly and immunocompromised adults, including patients with diabetes mellitus, alcoholism, and cancer (Pimentel et al. 2016).

Pathogenesis of $S$. agalactiae in humans is associated with the bacteria's ability to invade and pass through anatomic barriers such as vaginal or cervical epithelium. The mechanisms of $S$. agalactiae attachment, invasion, and translocation were investigated in a variety of cellular systems of human origin, including endothelial cells

doi: 10.1590/0074-02760170421

Financial support: FAPERJ, CAPES, CNPq, Sub-Reitoria de Pós-Graduação e

Pesquisa da Universidade do Estado do Rio de Janeiro (SR-2/UERJ).

+Corresponding author: pnagao@uol.com.br/pnagao@uerj.br

Received 6 October 2017

Accepted 5 March 2018
(Buscetta et al. 2016). However, further studies related to the molecular level of pathogen-host cell interactions are required. It is well known that modulation of actin microfilaments is critical for $S$. agalactiae invasion.

Phosphoinositide 3-kinase (PI3K) activation also occurs during the invasive process in host cells by $S$. agalactiae (Burnham et al. 2007). The formation of phosphatidylinositol 3,4,5-triphosphate ( $\mathrm{PIP}_{3}$ ) by PI3K leads to Akt phosphorylation and activation on the host-cell membrane. Manipulation of the PI3K/Akt pathway by a pathogen results in coordination of actin rearrangement, which leads to internalisation of the organism. The involvement of PI3K has been observed during the invasion process of $S$. agalactiae and other human pathogens (Burnham et al. 2007, Dowd et al. 2016).

In a previous study, our group showed that the interaction between $S$. agalactiae and human umbilical vein endothelial cells (HUVECs) induced the overexpression of two phosphotyrosine proteins, described as glutathione-S transferase (GST) and annexin V (Santos et al. 2009). Studies have shown that GST protects cells in up to $90 \%$ of cases of damage induced by oxidative stress (Awasthi et al. 2017). 
Reactive oxygen species (ROS) production by endothelial cells modulates redox-sensitive signalling mechanisms and gene expression that may also induce the activation of the PI3K/Akt pathway (Yang et al. 2017). The major source of ROS in endothelial cells is NADPH oxidase, an $\mathrm{O}_{2}$-generating enzyme also expressed in other mammalian cell types ( $\mathrm{Li}$ and Shah 2003). NADPH oxidase activation (oxidative burst) is essential for immune responses against infection (Ushio-Fukai 2006). A previously published study demonstrated that respiratory oxidative burst was triggered during $S$. agalactiae adherence to macrophages by the activation of NADPH oxidase (Teixeira et al. 2001).

Similarities in the NADPH oxidase complex have been demonstrated between endothelial cells and phagocytes ( $\mathrm{Li}$ and Shah 2002, Meijles et al. 2014). NADPH oxidase is a multicomponent enzyme that includes two membrane-bound components, gp91phox (also known as Nox2) and p22phox, and cytosolic subunits, including p40phox, p47phox, p67phox, and the small GTPase Rac1/2 (Abid et al. 2007, Lambeth et al. 2007). Among these subunits, p47phox is one of the most important regulators of NADPH oxidase activity. The cytoskeleton of host cells may play a role in the translocation and consequent activation of NADPH oxidase (Touyz et al. 2005, Meijles et al. 2014). However, the molecular mechanisms that promote ROS generation due to $S$. agalactiae infection in human endothelial cells remain unclear. To investigate the influence of oxidative stress on $S$. agalactiae-endothelial cell interactions, we focused on ROS production by activation of the p47phox NADPH oxidase subunit and the PI3K/Akt pathway during $S$. agalactiae-endothelial cell interaction.

\section{MATERIALS AND METHODS}

Bacterial strain origin and culture conditions - $S$. agalactiae capsular type III [GBS90356 cerebrospinal fluid (CSF) strain] isolated from a 3-day-old male baby with fatal acute meningitis that was partially investigated for virulence properties (Teixeira et al. 2001, Santos et al. 2009, Costa et al. 2011) was used in this study. For experiments described below, the GBS90356 strain was cultured on blood agar base (BAB; Oxoid) plates containing 5\% defibrinated sheep's blood for 24 $\mathrm{h}$ at $37^{\circ} \mathrm{C}$ and then grown in brain heart infusion broth (BHI; Difco) at $37^{\circ} \mathrm{C}$ until reaching an $\mathrm{OD}_{540}$ of $0.4\left[\sim 10^{8}\right.$ colony forming units (CFU) per $\mathrm{mL}$ ] (Santos et al. 2009).

S. agalactiae-HUVEC interaction assays - Primary HUVECs were obtained by treating umbilical veins with a $0.1 \%$ collagenase IV (Sigma) solution as previously described (Jaffe et al. 1973). All experiments were performed at least three times and each experiment was performed with a pool of HUVECs obtained from different donors. HUVECs were seeded onto $25-\mathrm{cm}^{2}$ bottles coated with porcine skin gelatine (Sigma) and grown in 199 medium (M199 - Sigma) supplemented with antibiotics, $100 \mathrm{U} / \mathrm{mL}$ penicillin, $100 \mu \mathrm{g} / \mathrm{mL}$ streptomycin, and 2.5 $\mu \mathrm{g} / \mathrm{mL}$ amphotericin-B, $2 \mathrm{mM}$ glutamine, and $20 \%$ foetal bovine serum at $37^{\circ} \mathrm{C}$ in a humidified $5 \% \mathrm{CO}_{2}$ atmosphere until they reached confluence. HUVECs grown in M199 without serum (18-24 h) were also used as negative control. Confluent HUVEC monolayers were used during first or second passages only, and subcultures were obtained by treatment with $0.025 \%$ trypsin $/ 0.2 \%$ EDTA solution prepared in $0.01 \mathrm{M}$ phosphate buffered $0.15 \mathrm{M} \mathrm{NaCl}$ at $\mathrm{pH} 7.2$ (PBS), rinsed in serum-depleted culture medium, and used for experiments in 24-well culture plates (Corning, NY, USA) (Santos et al. 2009).

HUVEC monolayers were pre-treated with or without diphenylene iodonium (DPI; $10 \mu \mathrm{M}$ ), an inhibitor of the catalytic subunit of NADPH oxidase or with apocynin $(10 \mu \mathrm{M})$, a compound that prevents coupling between p47phox and the corresponding catalytic subunit, or LY294002, a PI3K inhibitor $(5 \mu \mathrm{M})$ for $15 \mathrm{~min}$. Then, HUVECs were allowed to interact with $S$. agalactiae $\left(5 \times 10^{7} \mathrm{CFU}\right)$ for various time periods $(15,30,60,120$, and $180 \mathrm{~min}$ ) in $5 \% \mathrm{CO}_{2}$ at $37^{\circ} \mathrm{C}$. Subsequently, infected HUVECs were rinsed three times with PBS and lysed with $0.5 \mathrm{ml}$ of $25 \mathrm{mM}$ Tris, $5 \mathrm{mM}$ EDTA, $150 \mathrm{mM} \mathrm{NaCl}$, and $1 \%$ IGEPAL (lysis buffer). The total number of viable bacteria associated (intracellular plus extracellular) to HUVECs was estimated by determining the $\mathrm{CFU} / \mathrm{mL}$ on blood agar medium. Quantitative determination of intracellular bacteria was performed by a gentamicin-penicillin protection assay, as previously described (Santos et al. 2009). After each incubation period, the infected monolayers were incubated for an additional 2-h period in M199 containing $100 \mu \mathrm{g} / \mathrm{mL}$ gentamicin and $5 \mu \mathrm{g} / \mathrm{mL}$ penicillin G. The number of internalised viable bacteria was assessed as outlined above. Adherence rates were calculated as follows: [CFU of total cell-associated (internalised plus surface adherent) S. agalactiae - CFU internalised S. agalactiae] (Santos et al. 2009, Costa et al. 2011).

Measurement of intracellular ROS - HUVECs were incubated overnight on 96-well black-walled plates at a density of $6 \times 10^{3}$ cells $/$ well, at $37^{\circ} \mathrm{C}$ in a humidified atmosphere of $5 \% \mathrm{CO}_{2}$. The cells were washed three times with PBS and incubated with the probe CM-H2DCFDA [5-(and-6)-chloromethyl-2',7'dichlorodihydrofluorescein diacetate] for $30 \mathrm{~min}$. Cells were then washed with PBS and pre-treated with or without either an inhibitor of NADPH oxidase [(DPI; $10 \mu \mathrm{M}$ ) or apocynin $(10 \mu \mathrm{M})]$, the PI3K inhibitor LY294002 (5 $\mu \mathrm{M})$, or TNF- $\alpha(10 \mathrm{ng} / \mathrm{mL}$; positive control) and incubated in the absence or presence of S. agalactiae. Endothelial cells were serum-starved for 18-24 h to exclude serum-dependent effects as a negative control. Fluorescence intensity was assessed throughout the 180-min period using an Envision microplate reader. ROS production was detected through fluorescence emitted from dichlorofluorescein (DCF) oxidation. Fluorescence was monitored at excitation and emission wavelengths of 495 $\mathrm{nm}$ and $525 \mathrm{~nm}$, respectively (Ribeiro-Pereira et al. 2014).

Real time quantitative reverse transcription polymerase chain reaction (Real-time qRT-PCR) - Total RNA was isolated from samples obtained from HUVEC controls or those infected with $S$. agalactiae by using an RNeasy Mini Kit (Qiagen, Hilden, Germany) according to the manufacturer's instructions. The isolated mRNA was transcribed into cDNA using SuperScript III 
First-Strand (Invitrogen, Carlsbad, CA) according to the manufacturer's protocol. The primers used for $\mathrm{p} 47$ phox were 5'-CCCTGCTGGGCTTTGAGAA-3' (sense) and 5'-CCGACAGGTCCTGCCATTT-3' (antisense) (Watanabe et al. 2003). GAPDH was used as the housekeeping gene (endogenous control) for normalisation and was amplified using the following primers: 5'-TGCACCACCAACTGCTTAGC-3' (sense) and 5'-GCCATGGACTGTGGTCATGAG-3' (antisense) (Shen et al. 2013). Real Time qRT-PCR was performed using GoTaq qPCR Master Mix reagents (Promega) into a 7500 real-time PCR system thermocycler (Applied Biosystems, Foster City, CA). All real-time PCR reactions were performed in triplicate for each independent experiment. Data were normalised to GAPDH expression and relative expression was calculated by the 2- $\Delta \Delta$ CT comparative method (Bülbül et al. 2014).

Immunoblot analysis - HUVEC monolayers were infected with $S$. agalactiae for different time periods as described above. Following infection, the plates were chilled, and all subsequent steps were carried out at $4^{\circ} \mathrm{C}$ as previously described (Costa et al. 2016). The HUVECs were then rinsed with PBS containing $0.4 \mathrm{mM} \mathrm{Na}_{3} \mathrm{VO}_{4}$ and $1 \mathrm{mMNaF}$ per $\mathrm{mL}$. Next, the infected cells were scraped from the plate, resuspended in $1.5 \mathrm{~mL}$ of the same buffer solution, collected by centrifugation for $1 \mathrm{~min}$ at 12,000 ' $\mathrm{g}$, and lysed for $30 \mathrm{~min}$ in $100 \mu \mathrm{L}$ of $50 \mathrm{mM}$ Tris$\mathrm{HCL}$ ( $\mathrm{pH}$ 7.6) containing $0.4 \mathrm{mM} \mathrm{Na}_{3} \mathrm{VO}_{4}, 1 \mathrm{mM} \mathrm{NaF}$, $1 \%$ Triton X-100, $100 \mu \mathrm{M}$ of phenylethylsulfonyl fluoride, $40 \mu \mathrm{M}$ of leupeptin, and $2 \mathrm{mM}$ EDTA. The proteins were then quantified, and $30 \mu \mathrm{g}$ of protein from each extract was subjected to electrophoresis in $12 \%$ polyacrylamide separating gel (SDS-PAGE). Proteins were transferred to nitrocellulose membranes (BioRad), which were blocked and then incubated with polyclonal anti-human antibodies: phospho-p47phox, $\beta$-actin, total Akt, and phosphoAkt. The membranes were incubated with the appropriate peroxidase-conjugated secondary antibody and immunoreactivity was detected using an ECL Plus detection kit
(Amersham Biosciences, Buckinghamshire, UK). Autoradiographs were quantified by scanning densitometry, and the resulting absorbance curves were integrated using the Scion Image Master. Densitometric analyses were performed on gels with different exposure times, and the ones giving linear absorbance curves were used for semiquantitative assessment (Santos et al. 2013).

Immunofluorescence - HUVECs were cultured on glass coverslips then pre-treated or not with DPI (10 $\mu \mathrm{M})$, apocynin $(10 \mu \mathrm{M})$, or LY294002 $(5 \mu \mathrm{M})$ for $15 \mathrm{~min}$ and then allowed to interact with fluorescein-isothiocyanate (FITC)-labelled S. agalactiae for $60 \mathrm{~min}$ at $37^{\circ} \mathrm{C}$. The cells were washed with PBS, fixed in $3.7 \%$ formaldehyde for $10 \mathrm{~min}$ at $25^{\circ}$ and washed again 3 times with PBS-BSA (10 min each). Cells were permeabilised with $0.1 \%$ Triton X-100 in PBS for 6 min, washed, and stained with $0.1 \mu \mathrm{g} / \mathrm{mL}$ fluorescein isothiocyanate-phalloidin, $0.5 \mu \mathrm{g} / \mathrm{mL}$ 4'-6-diamidino-2-phenylindole (DAPI) or anti-phosphorylated $p 47 p h o x$ Alexa 546 conjugated for 30 min. Cells were visualised in an Olympus Microscope Model IX71 TH4-100 (Santos et al. 2009).

Statistical analysis - The values of different treatments were compared using Student's $t$-test and ANOVA, followed by Bonferroni's $t$-test for unpaired values. All statistical analyses were performed at the $p<0.05$ level of significance.

\section{RESULTS}

ROS production during S. agalactiae-HUVEC interaction - Levels of ROS generation were evaluated during $S$. agalactiae-HUVEC interaction using inhibitors of NADPH oxidase (DPI and apocynin) and PI3K (LY294002). Non-infected HUVEC monolayers treated with TNF- $\alpha$ were used as positive controls. Increased ROS production was observed in HUVECs after $15 \mathrm{~min}$ of infection (Fig. 1; $<<0.02$ ). Moreover, pre-treatment of HUVEC with all inhibitors decreased ROS production during all time periods $(\mathrm{p}<0.03)$. HUVECs grown in

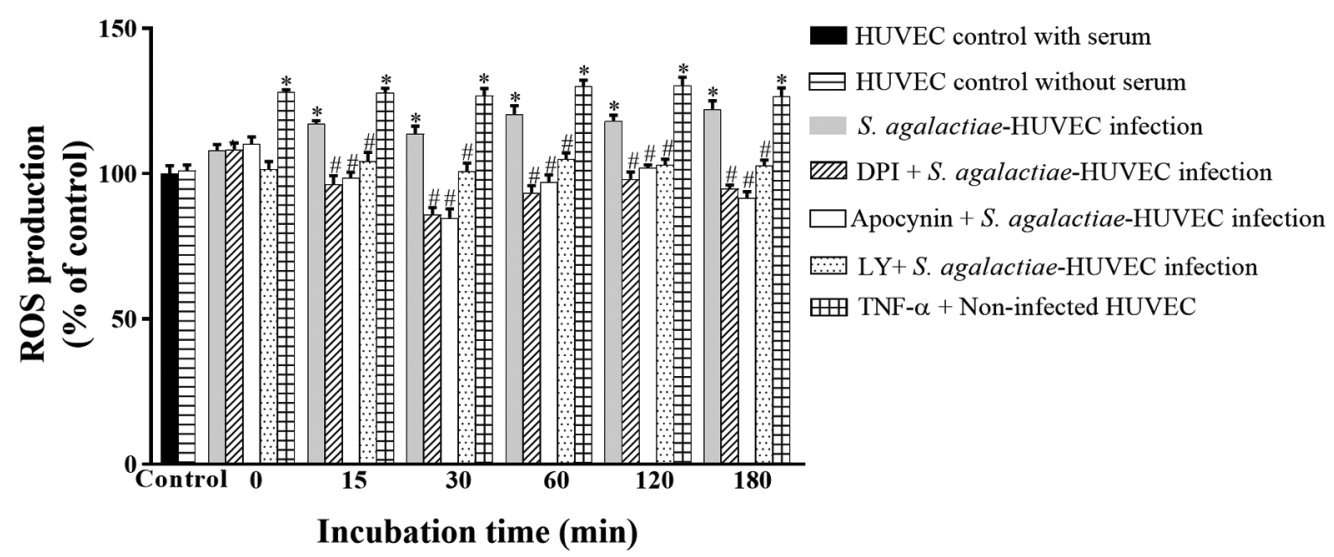

Fig. 1: reactive oxygen species (ROS) generation by NADPH oxidase activity during infection of human primary endothelial cells (HUVECs) by Streptococcus agalactiae. Experiments were performed at different periods of incubation using the GBS90356 type III strain of $S$. agalactiae and HUVECs in the presence or absence of NADPH inhibitors (10 $\mu \mathrm{M}$ DPI, $10 \mu \mathrm{M}$ apocynin) or a PI3K inhibitor $(5 \mu \mathrm{M}$ LY294002). Non-infected HUVEC monolayers with and without serum were used as negative controls. Non-infected HUVEC monolayers with serum and $10 \mathrm{ng} / \mathrm{mL}$ TNF- $\alpha$ were used as positive controls. Data are expressed as mean \pm SD of three independent experiments; *p $\leq$ 0.05 vs non-infected HUVECs; $\# \mathrm{p} \leq 0.05$ vs $S$. agalactiae-HUVEC infection. 
serum starvation conditions for $18-24$ h to exclude serum-dependent effects were used as a negative control.

Activation of p47phox NADPH oxidase subunit during S. agalactiae-HUVEC interaction - Results of immunofluorescence assays of p47phox activity during ROS generation by $S$. agalactiae infection of HUVEC were demonstrated in Fig. 2A-F. The NADPH oxidase p47phox subunit was mostly observed near the nucleus of uninfected host cells (Fig. 2A). HUVECs infected with $S$. agalactiae showed changes in subcellular localisation of the p47phox subunit, which was previously shown to be central for even distribution in the cell, reflecting activation of p47phox in HUVECs (Fig. 2B). Additional assays are required to elucidate mechanisms involved in $\mathrm{p} 47 \mathrm{phox}$ activation by $S$. agalactiae. DPI and apocynin inhibition assays (Fig. 2C-F) were performed at $60 \mathrm{~min}$ post-infection when the highest adherence level to HUVECs by $S$. agalactiae was observed. Treatment with both inhibitors resulted in higher adherence levels of $S$. agalactiae 60 min post-infection of in HUVECs (Fig. 2D, F).
NADPH oxidase p47phox expression during S. agalactiae-HUVEC - Data displayed in Fig. 3A show an increase of p47phox mRNA expression at $30 \mathrm{~min}$ and 60 min post-infection, as measured by Real Time qRT-PCR $(\mathrm{p}<0.03)$. Immunoblotting assay results confirmed higher $\mathrm{p} 47$ phox activities at upon $15 \mathrm{~min}$ and $60 \mathrm{~min}$ incubation of S. agalactiae-HUVEC ( $<$ < 0.04) (Fig. 3B, C).

Effect of DPI and Apocynin on activation of the p47phox NADPH oxidase subunit during S. agalactiaeHUVEC interaction - The influence of the NADPH oxidase p47phox subunit on $S$. agalactiae adherence to and intracellular viability of HUVEC using inhibitors of p47phox (DPI or apocynin) are displayed in Fig. 4. A time-dependent increase in adherence to HUVECs was observed upon treatment with both inhibitors. A higher number of adherent bacteria was observed in HUVECs pre-treated with DPI or apocynin, compared to that of untreated HUVECs $(\mathrm{p}<0.03)$ (Fig. 4A). A significant decrease in the number of internalised bacteria occurred in HUVECs treated with DPI and apocynin af-
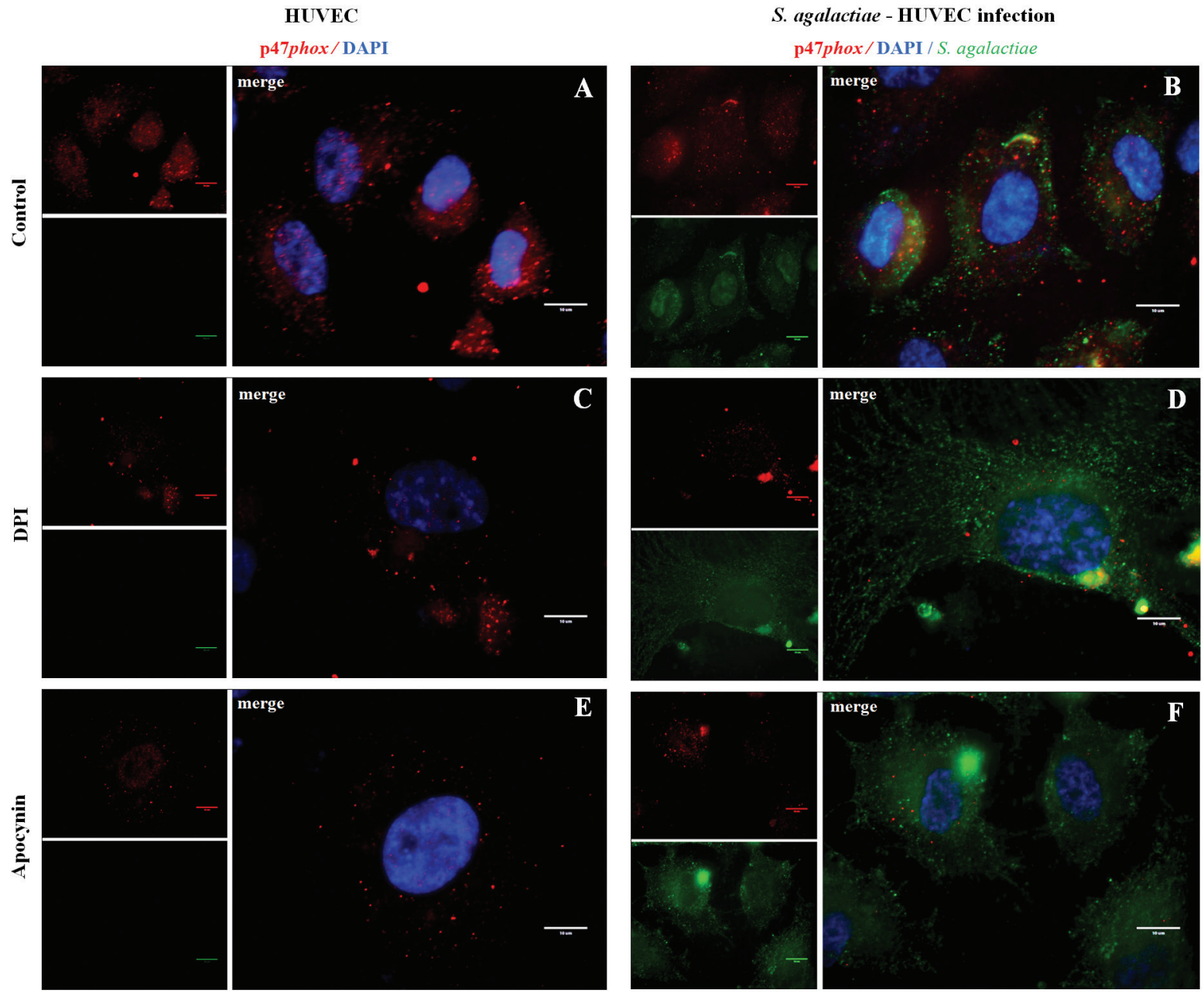

Fig. 2: immunofluorescence assays of NADPH oxidase p47phox subunit activity during infection of human primary endothelial cells (HUVECs) by Streptococcus agalactiae. (A, C, E) Uninfected HUVECs and (B, D, F) HUVECs infected by S. agalactiae in the presence or absence of NADPH inhibitors (10 $\mu \mathrm{M}$ DPI, $10 \mu \mathrm{M}$ apocynin). Anti-p47phox was labelled with Alexa 546 (red), S. agalactiae with fluorescein-isothiocyanate (FITC) (green), and the nuclei were stained with DAPI (blue). Scale bars, $10 \mu \mathrm{M}$. 
A

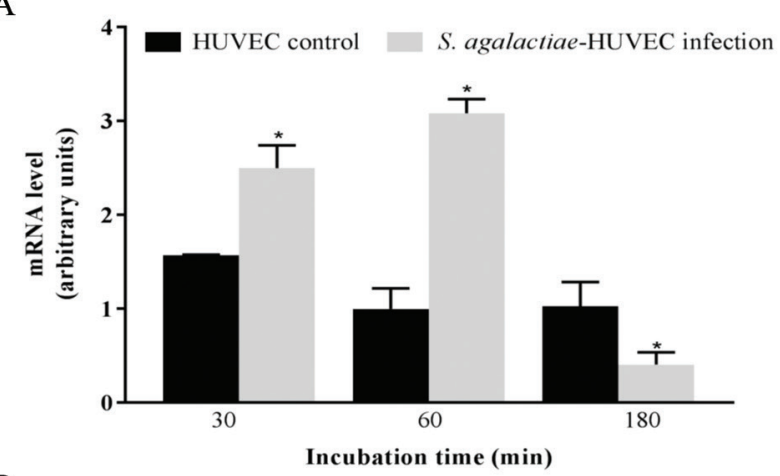

B

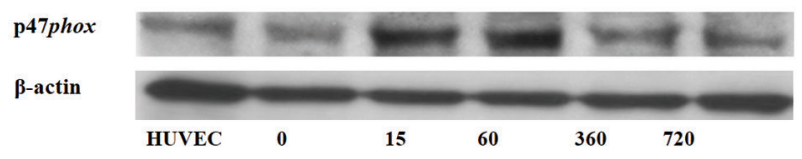

$\mathrm{C}$

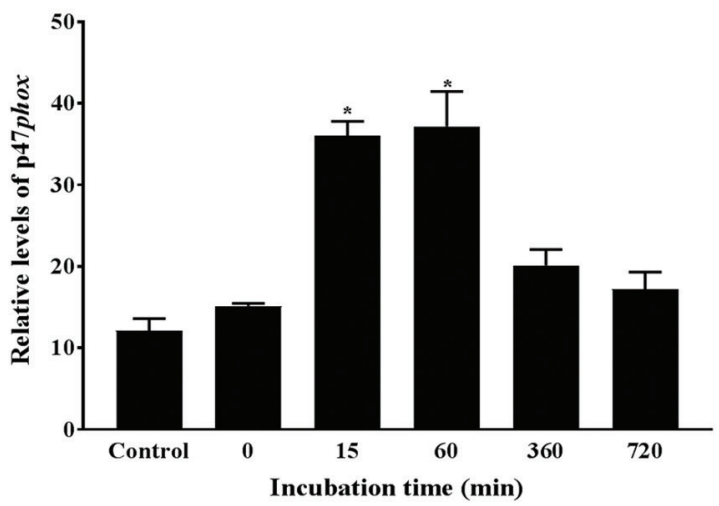

Fig. 3: expression of NADPH oxidase p47phox subunit during infection of HUVECs with Streptococcus agalactiae. Data were analysed at different periods of time by (A) real time quantitative reverse transcription polymerase chain reaction (real-time qRT-PCR), (B) immunoblotting, and $(\mathrm{C})$ densitometry assays. Actin was used as a control for protein loading. Data are expressed as mean $\pm \mathrm{SD}$ of three experiments; ${ }^{*} \mathrm{p}<0.05$.

ter the first 30 min of infection compared to the number in untreated HUVECs (Fig. 4B).

Involvement of the PI3K/Akt pathway during $S$. agalactiae-HUVEC interaction - Data shown in Fig. 5A-B demonstrate the effect of the PI3K/Akt pathway on the interaction between $S$. agalactiae and HUVECs pre-treated with the PI3K inhibitor, LY294002. A timedependent increase in bacterial adherence was observed when HUVECs were treated with LY294002, similarly to results achieved with p47phox (DPI and apocynin) inhibitors. The highest adherence level was observed at 120 min post-infection $(\mathrm{p}<0.02)$ (Fig. 5A). Conversely, bacterial internalisation was detected at lower levels from 30 min to 180 min post-infection ( $<$ 0.01) (Fig. 5B).

Immunofluorescence staining assays illustrated the involvement of PI3K/Akt pathway activation in the actin stress fibre assembly of endothelial cells upon by $S$. agalactiae infection (Fig. 5C-D). Similar to non-infected HUVECs, pre-treatment with NADPH oxidase (DPI and apocynin) or PI3K (LY294002) inhibitors in the presence (Fig. 5F, H, J) or absence of $S$. agalactiae infection (Fig. $5 \mathrm{E}, \mathrm{G}, \mathrm{I})$ did not induce actin stress fibre arrangement. As shown in Fig. 5K, immunoblotting results revealed higher levels of phosphorylated Akt expression at $15 \mathrm{~min}$ and 30 min post-infection of HUVECs by $S$. agalactiae. Inhibition assays with LY294002 (15 min and $30 \mathrm{~min}$ post-infection) confirmed the involvement of the PI3K/ AKT pathway during $S$. agalactiae internalisation in endothelial cells. Results were confirmed by densitometry analysis (data not shown).

\section{DISCUSSION}

ROS have been classically regarded as host defence molecules related to destroying exogenous human pathogens. Also, it has long been recognised that increased ROS levels modify cell signalling of host proteins, leading to pathological processes such as inflammation and bacterial infections. Nevertheless, accumulated evidence indicates that ROS are second messengers and cell signalling modifiers (Zhang et al. 2016).

Only a few studies concerning NADPH oxidase activation during Streptococcus spp. infection were found in the available literature. For Streptococcus pyogenes, the production of $\mathrm{O}_{2}^{-}$was found as the result of NADPH oxidase induction during bacterial infection in human

$\mathbf{A}$
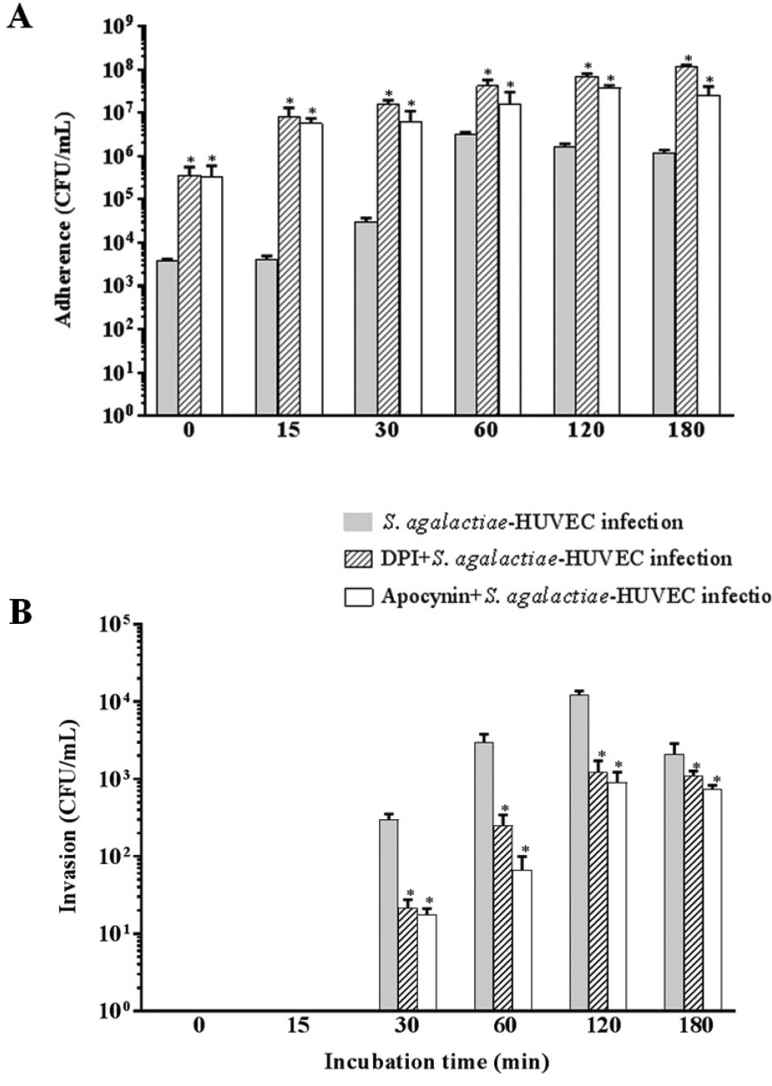

Fig. 4: influence of NADPH oxidase p47phox subunit on Streptococcus agalactiae-human primary endothelial cells (HUVECs) interaction. (A) Adherence to and (B) intracellular viability of $S$. agalactiae in HUVECs pre-treated with DPI $(10 \mu \mathrm{M})$ or apocynin $(10 \mu \mathrm{M})$ to inhibit p47phox. Data are expressed as mean \pm SD of three experiments; ${ }^{*} \mathrm{p}<0.05$. 
A

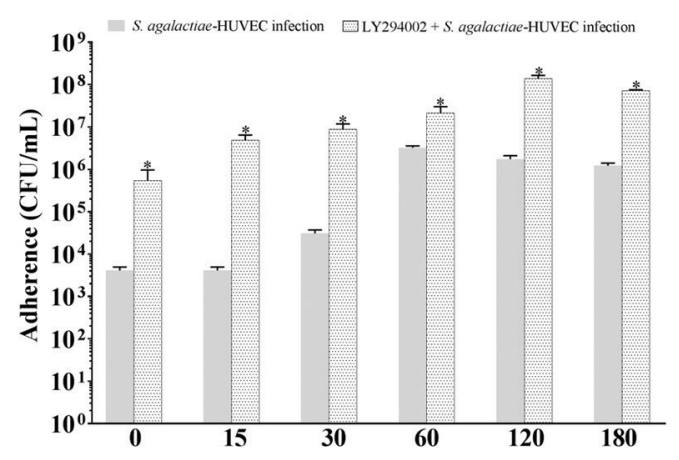

B

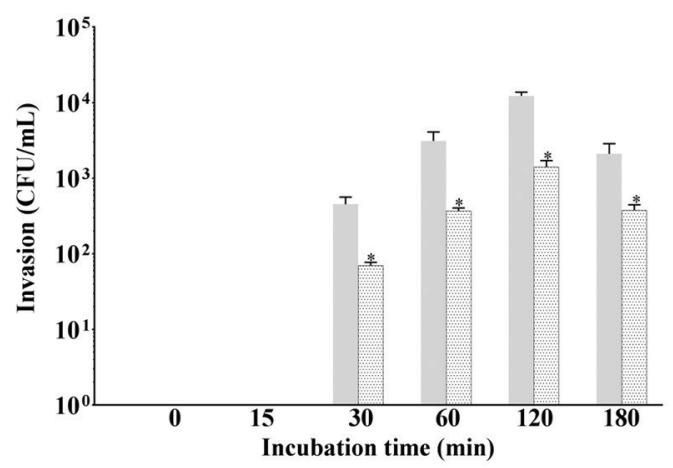

K

S. agalactiae

LY 294002

PAKT

AKT total

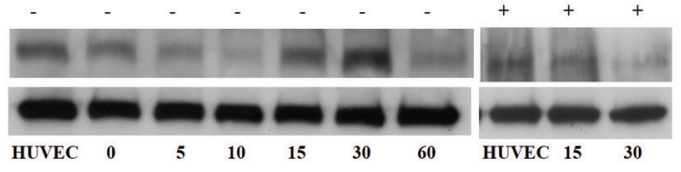

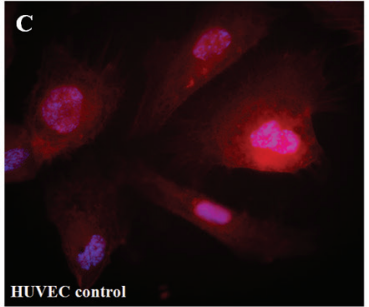
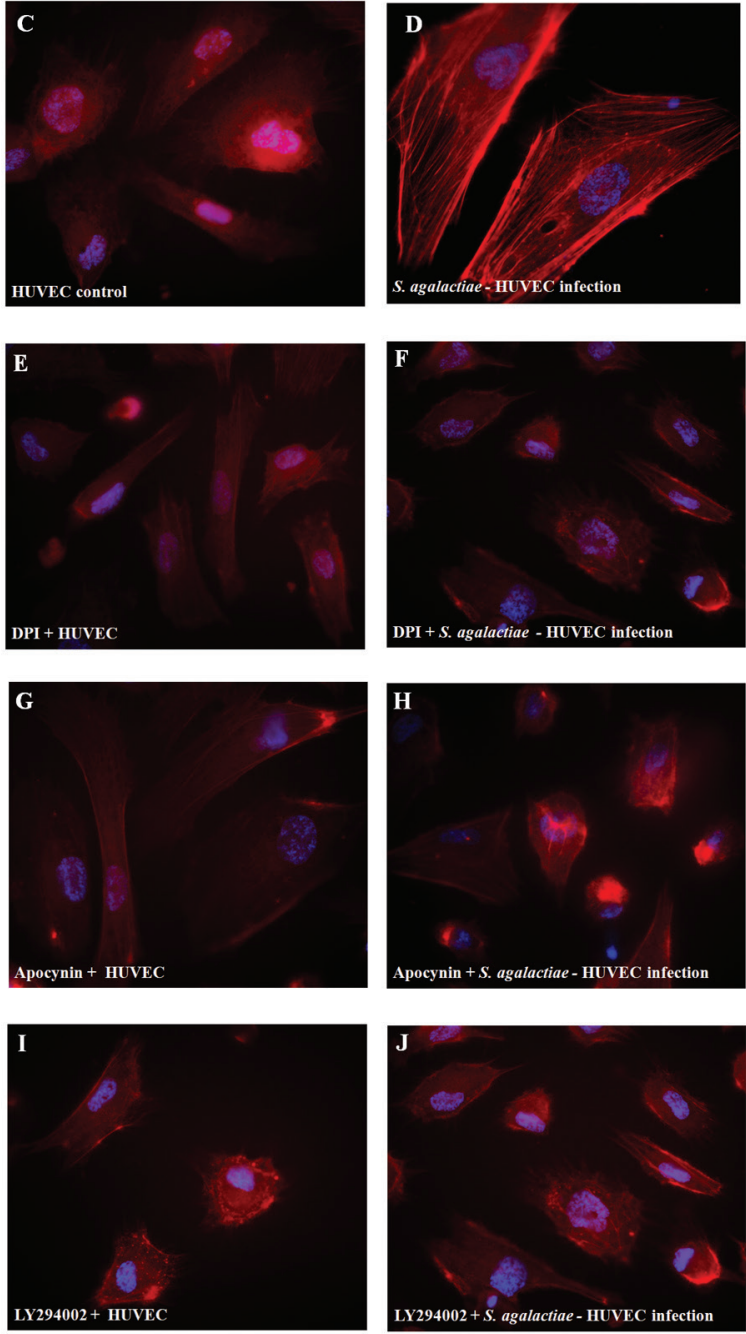

Fig. 5: influence of the PI3K/Akt pathway on the Streptococcus agalactiae-human primary endothelial cells (HUVECs) interaction. (A) Adherence to and (B) invasion of S. agalactiae in HUVECs pre-treated with LY294002, an inhibitor of PI3K. Micrographic images of immunofluorescence staining indicated: (C) non-infected HUVEC monolayers as a negative control; (D) generation of actin stress fibres in HUVECs infected with S. agalactiae; (E, G, I) HUVECs pre-treated with DPI (10 $\mu$ M), apocynin (10 $\mu$ M), and LY294002 (5 $\mu \mathrm{M})$, respectively; (F, H, J) HUVECs pre-treated with inhibitors (DPI, apocynin and LY294002, respectively) and infected with $S$. agalactiae. (K) Immunoblotting assay of phosphorylated Akt in infected HUVECs pre-treated with LY294002. Data are expressed as mean \pm SD of three experiments. *p $<0.05$.

primary keratinocytes (Regnier et al. 2016). The effect of NADPH oxidase inhibitor (DPI) treatment on intracellular survival of $S$. pyogenes during the early stage of phagocytosis by HL-60 cells was also verified (Nordenfelt et al. 2009). Moreover, the ability of S. agalacti$a e$ to trigger oxidative burst in murine macrophages by NADPH oxidase activation was also demonstrated (Teixeira et al. 2001). In addition, the role of $\mathrm{p} 47$ phox in functionally active NADPH oxidase activity was evidenced by using p47phox ${ }^{-/}$mice assays (Li and Shah 2002, Touyz et al. 2005). Similar effects were demonstrated for endothelial cells: the major source of ROS was also described as NADPH oxidase, whose activation and regulation were controlled by the phosphorylation of its cytosolic component p47phox (Schuett et al. 2017). However, physiological substrates, regulation, and control of signalling networks, especially of endothelial cells, need further investigation.
Presently, the involvement of p47phox subunit in $S$. agalactiae-HUVEC interaction was demonstrated by Real Time qRT-PCR, western blotting, and immunofluorescence assays. ROS production by activation of the p47phox NADPH oxidase subunit during $S$. agalactiaeHUVEC interaction was demonstrated. Thus, our results agree with those of Zhang et al. (2016) showing that DPI and apocynin reduce $\mathrm{p} 47$ phox translocation and expression, thereby suppressing ROS production in peripheral blood mononuclear cells from premature infants. Similarly, Streptococcus pneumoniae induces a higher oxidative burst in neutrophils by triggering neutrophil NADPH oxidase to produce more reactive oxygen intermediates and by activating $\mathrm{p} 47$ phox by $S$. pneumoniae in the plasma membrane fraction of neutrophils (Barbuti et al. 2010).

It is well-known that $S$. agalactiae needs cytoskeleton reorganisation to invade host cells (Boone 
et al. 2012). Interestingly, previous studies have also reported the involvement of ROS in cytoskeletal reorganisation accompanying $\mathrm{O}_{2}^{-}$formation acting as a scaffold for the p47phox subunit (Touyz et al. 2005). A close relationship between actin polymerisation and ROS generation has also been observed after wounding endothelial cell monolayers (Li and Shah 2002).

The PI3K/Akt signalling pathway was found to be associated with cytoskeletal regulation, vesicle trafficking, and the balance between cellular survival and regulated cell death (Burnham et al. 2007). PI3K activation has been specifically implicated in modification and manipulation of the actin cytoskeleton, essentially in $S$. agalactiae invasion of host cells (Burnham et al. 2007). The involvement of the PI3K/Akt pathway during the $S$. agalactiae-HUVEC interaction was verified in the present study. Burnham et al. (2007) showed an inhibition of $S$. agalactiae invasion in HeLa cells treated with the LY294002 inhibitor of PI3K. Similar to previous results of these authors, current data concerning the inhibition of ROS production showed a decrease in invasion and an increase in the adhesion levels of $S$. agalactiae on HUVEC induced by LY294002. Data indicated that $S$. agalactiaeHUVEC interaction was partially due to alterations in the organisation of microfilaments of the host cells through the PI3K/Akt pathway. As soon as S. agalactiae began to establish physical contact with the host cell surface, the formation of stress fibres was observed in HUVECs. Additional results demonstrated loss of stress fibres due to inhibition of PI3K, reinforcing the participation of the PI3K/Akt pathway in human endothelial cells.

The ability of Akt to regulate multiple activities of host cells makes it an essential target for invading bacterial pathogens, including $S$. agalactiae. A previous study in HeLa cells indicated that Akt was required for efficient $S$. agalactiae invasion, since Akt was rapidly phosphorylated in response to $S$. agalactiae (Burnham et al. 2007). In our work using HUVECs, a significant increase in Akt phosphorylation occurred at 15-30 min post-infection of $S$. agalactiae, suggesting the involvement of PI3K/Akt in this process.

In conclusion, ROS generation via the NADPH oxidase pathway in human endothelial cells accompanied by cytoskeletal reorganisation through the PI3K/Akt pathway occurred during invasion of the $S$. agalactiae GBS90356 strain isolated from a fatal case of meningitis. These results may contribute to the understanding of virulence mechanisms involved in the translocation of $S$. agalactiae across the blood-brain barrier.

\section{AUTHORS' CONTRIBUTION}

JSSO, GSS and PEN - Conception of the general project and experimental design; JAM and AMS performed molecular techniques and reviewed the experimental results; JSSO and GSS - performed immunoblot analysis and immunofluorescence assays; TCBF - provided reagents and assistance in drafting the manuscript; PEN and ALMG - analysis and discussion of the results, assistance in creating the figures, literature review, and preparation of the manuscript.

\section{REFERENCES}

Abid MR, Spokes KC, Shih SC, Aird WC. NADPH oxidase activity selectively modulates vascular endothelial growth factor signaling pathways. J Biol Chem. 2007; 282(48): 35373-85.

Awasthi YC, Ramana KV, Chaudhary P, Srivastava SK, Awasthi S. Regulatory roles of glutathione-S-transferases and 4-hydroxynonenal in stress-mediated signaling and toxicity. Free Radic Biol Med. 2017; 111: 235-43.

Barbuti G, Moschioni M, Fumarulo R, Censini S, Montemurro P. Streptococcus pneumoniae modulates the respiratory burst response in human neutrophils. FEMS Immunol Med Microbiol. 2010; 60(1): 57-62.

Boone TJ, Tyrrell GJ. Identification of the actin and plasminogen binding regions of group B streptococcal phosphoglycerate kinase. J Biol Chem. 2012; 287(34): 29035-44.

Bülbül N, Pala E, İğci YZ, Göğebakan B, Öztuzcu S, Cengiz B, et al. NADPH oxidase p22phox gene expression in ulcerative colitis. Turk J Gastroenterol. 2014; 25(6): 634-8.

Burnham CD, Shokoples SE, Tyrrell GJ. Invasion of HeLa cells by group B Streptococcus requires the phosphoinositide-3-kinase signaling pathway and modulates phosphorylation of host cell Akt and glycogen synthase kinase-3. Microbiology. 2007; 153(Pt 12): 4240-52.

Buscetta M, Firon A, Pietrocola G, Biondo C, Mancuso G, Midiri A, et al. $\mathrm{PbsP}$, a cell wall-anchored protein that binds plasminogen to promote hematogenous dissemination of Group B Streptococcus. Mol Microbiol. 2016; 101(1): 27-41.

Costa AF, Moraes JA, de Oliveira JS, dos Santos MH, Santos GS, Barja-Fidalgo C, et al. Reactive oxygen species involved in apoptosis induction of human respiratory epithelial (A549) cells by Streptococcus agalactiae. Microbiology. 2016; 162(1): 94-9.

Costa AF, Pereira CS, Santos GS, Carvalho TM, Hirata Jr R, de Mattos-Guaraldi AL, et al. Group B Streptococcus serotypes III and $\mathrm{V}$ induce apoptosis and necrosis of human epithelial A549 cells. Int J Mol Med. 2011; 27(5): 739-44.

Dowd GC, Bhalla M, Kean B, Thomas R, Ireton K. Role of Host Type IA phosphoinositide 3-kinase pathway components in invasinmediated internalization of Yersinia enterocolitica. Infect Immun. 2016; 84(6): 1826-41.

Jaffe EA, Nacchman RL, Becker CG, Minick CR. Culture of human endothelial cells of derivate from umbilical cords veins. Identification on by morphologic and immunologic criteria. J Clin Invest. $1973 ;$ 52(11): 2745-56.

Lambeth JD, Kawahara T, Diebold B. Regulation of Nox and Duox enzymatic activity and expression. Free Radic Biol Med. 2007; 43(3): 319-31.

Li J, Shah AM. Intracellular localization and preassembly of the NADPH oxidase complex in cultured endothelial cells. J Biol Chem. 2002; 277(22): 19952-60.

Li JM, Shah AM. Mechanism of endothelial cell NADPH oxidase activation by angiotensin II. Role of the p47phox subunit. J Biol Chem. 2003; 278(14): 12094-100.

Meijles DN, Fan LM, Howlin BJ, Li JM. Molecular insights of p47phox phosphorylation dynamics in the regulation of NADPH oxidase activation and superoxide production. J Biol Chem. 2014; 289(33): 22759-70.

Nordenfelt P, Bauer S, Lönnbro P, Tapper H. Phagocytosis of Streptococcus pyogenes by all-trans retinoic acid-differentiated HL-60 cells: roles of azurophilic granules and NADPH oxidase. PLoS One. 2009; 4(10): e7363. 
Pimentel BA, Martins CA, Mendonça JC, Miranda PS, Sanches GF, Mattos-Guaraldi AL, et al. Streptococcus agalactiae infection in cancer patients: a five-year study. Eur J Clin Microbiol Infect Dis. 2016; 35(6): 927-33.

Regnier E, Grange PA, Ollagnier G, Crickx E, Elie L, Chouzenoux S, et al. Superoxide anions produced by Streptococcus pyogenes group A-stimulated keratinocytes are responsible for cellular necrosis and bacterial growth inhibition. Innate Immun. 2016; 22(2): 113-23.

Ribeiro-Pereira C, Moraes JA, Souza MJ, Laurindo FR, Arruda MA, Barja-Fidalgo C. Redox modulation of FAK controls melanoma survival: role of NOX4. PLoS One. 2014; 9(6): e99481.

Santos GS, Penha CL, Mattos-Guaraldi A, Attias M, Lopes-Bezerra LM, Silva-Filho FC, et al. Group B Streptococcus induces tyrosine phosphorylation of annexin V and glutathione S-transferase in human umbilical vein endothelial cells. Int J Mol Med. 2009; 24(3): 393-9.

Santos MH, da Costa AF, Ferreira BJ, Souza SL, Lannes PS, Santos GS, et al. A phosphoramidon-sensitive metalloprotease induces apoptosis of human endothelial cells by Group B Streptococcus. Antonie van Leeuwenhoek. 2013; 104(6): 1125-33.

Schuett J, Schuett H, Oberoi R, Koch AK, Pretzer S, Luchtefeld M, et al. NADPH oxidase NOX2 mediates TLR2/6-dependent release of GM-CSF from endothelial cells. FASEB J. 2017; 31(6): 2612-24.

Shen Y, Fu W-Y, Cheng EYL, Fu AKY, Ip NY. Melanocortin-4 receptor regulates hippocampal synaptic plasticity through a protein kinase A-dependent mechanism. J Neurosci. 2013; 33(2): 464-72.
Teixeira CF, Azevedo NL, Carvalho TM, Fuentes J, Nagao PE. Cytochemical study of Streptococcus agalactiae and macrophage interaction. Microsc Res Tech. 2001; 54(4): 254-9.

Touyz RM, Yao G, Quinn MT, Pagano PJ, Schiffrin EL. p47phox associates with the cytoskeleton through cortactin in human vascular smooth muscle cells role in $\mathrm{NAD}(\mathrm{P}) \mathrm{H}$ oxidase regulation by angiotensin II. Arterioscler Thromb Vasc Biol. 2005; 25(3): 512-8.

Ushio-Fukai M. Localizing NADPH oxidase-derived ROS. Sci STKE. 2006; 2006(349): re8.

Verani JR, McGee L, Schrag SJ, Division of Bacterial Diseases, National Center for Immunization and Respiratory Diseases, Centers for Disease Control and Prevention (CDC). Prevention of perinatal group B streptococcal disease - revised guidelines from CDC, 2010. MMWR Recomm Rep. 2010; 59(RR-10): 1-36.

Watanabe H, Adachi R, Hirayama A, Kasahara T, Suzuki K. Triphenyltin enhances the neutrophilic differentiation of promyelocytic HL-60 cells. Biochem Biophys Res Commun. 2003; 306(1): 26-31.

Yang K, Zhang H, Luo Y, Zhang J, Wang M, Liao P, et al. Gypenoside XVII prevents atherosclerosis by attenuating endothelial apoptosis and oxidative stress: insight into the ER $\alpha$-Mediated PI3K/Akt pathway. Int J Mol Sci. 2017; 18(2): pii: E77.

Zhang L, Dong W, Li Q, Kang L, Zhang L, Lu Y, et al. Mechanism of p47phox-induced increase of reactive oxygen species in peripheral blood mononuclear cells from premature infants on oxygen therapy. J Matern Fetal Neonatal Med. 2016; 29(21): 3490-4. 